Chapter 4: Magnetic field structure and dynamics of flaring regions 


\title{
Nonlinear force-free modeling of magnetic fields in flare-productive active regions
}

\author{
M. S. Wheatland ${ }^{1}$ and S. A. Gilchrist ${ }^{2}$ \\ ${ }^{1}$ School of Physics, University of Sydney \\ NSW 2006 Australia \\ email: michael.wheatland@sydney.edu.au \\ ${ }^{2}$ NorthWest Research Associates \\ 3380 Mitchell Lane, Boulder, CO 80301-2245 USA \\ email: sgilchrist@nwra.com
}

\begin{abstract}
We review nonlinear force-free field (NLFFF) modeling of magnetic fields in active regions. The NLFFF model (in which the electric current density is parallel to the magnetic field) is often adopted to describe the coronal magnetic field, and numerical solutions to the model are constructed based on photospheric vector magnetogram boundary data. Comparative tests of NLFFF codes on sets of boundary data have revealed significant problems, in particular associated with the inconsistency of the model and the data. Nevertheless NLFFF modeling is often applied, in particular to flare-productive active regions. We examine the results, and discuss their reliability.
\end{abstract}

Keywords. Sun: activity, Sun: corona, Sun: flares, Sun: magnetic fields

\section{Introduction}

Sunspot magnetic fields power large scale solar activity, i.e. flares and coronal mass ejections (CMEs), which can produce space weather storms (e.g., Baker et al. 2008). Terrestrial space weather effects motivate the modeling of active region magnetic fields. Accurate models may assist with understanding and quantifying processes of magnetic energy storage and release, and may lead to improved event prediction.

A variety of models may be used to represent active region coronal magnetic fields. Magneto-hydrodynamics (MHD) provides a time-dependent model for the field $\mathbf{B}$, the fluid velocity $\mathbf{v}$, the fluid pressure $p$ and the fluid density $\rho$. Numerical solution of the MHD equations requires specification of initial values for the dependent variables, and boundary conditions on the variables at all times. The process is computationally intensive and requires detailed boundary conditions. The model is not generally applied directly to solar data (but see e.g., Amari, Canou \& Aly 2014). More often it is applied to prescribed boundary and initial configurations which resemble solar configurations, or else solar data are combined with synthetic boundary data in some way. Magnetohydrostatic modeling is a simpler, static approach, involving a boundary value problem for $\mathbf{B}, p$, and $\rho$. In principle this model may be useful for modeling from solar data, but it has not been widely used to date (but see e.g., Gilchrist \& Wheatland 2013; Wiegelmann, Petrie \& Riley 2015). The nonlinear force free field (NLFFF) model is a static model presenting a boundary value problem for the field $\mathbf{B}$ alone. Numerical solution of this model is less computationally intensive than MHD, and it is often applied to solar boundary data (photospheric vector magnetograms).

The NLFFF model has become popular, with dozens of papers a year presenting solutions to the model for solar data, and interpreting and using the results (an ADS search 
for abstracts containing the words "nonlinear", "force", "free" and "solar" returned 40 refereed papers for 2012, 46 papers for 2013, and 44 papers for 2014). A basic problem with NLFFF modeling is that the boundary data are in general inconsistent with the model, as highlighted by the comparisons of results for NLFFF methods presented in Schrijver et al. (2008), Metcalf et al. (2008), and DeRosa et al. (2009). This problem is rarely discussed in new papers using the model. Recently DeRosa et al. (2015) revisited the question of the reliability of NLFFF modeling in application to solar data, presenting results for a number of different NLFFF codes applied to vector magnetograms prepared with different spatial resolutions. $\dagger$

This paper presents a brief review of NLFFF modeling, including the solar data used, the details of the model and of the boundary conditions, and the problem of inconsistency and its effects on methods of solution of the model. Strategies for assessing the reliability of results are discussed.

\section{NLFFF modeling}

\subsection{Vector magnetogram data}

Vector magnetograms are maps of the photospheric vector magnetic field $\mathbf{B}=\left(B_{x}, B_{y}, B_{z}\right)$ in local cartesian heliographic coordinates, where $z$ is the local radial direction. The field values are constructed from observations of magnetically sensitive spectral lines formed close to the photosphere (del Toro Iniesta 2003). Stokes Polarimeters measure polarisation profiles $I(\lambda), Q(\lambda), U(\lambda), V(\lambda)$ as a function of wavelength $\lambda$ across spectral lines, for points within fields of view on the disk, or for the whole disk. The magnetic fields parallel to, and perpendicular to, the line of sight are obtained from the polarisation measurements by applying a radiative transfer model. This process is known as Stokes inversion. The vector components of the fields are obtained after resolution of the intrinsic 180 degree ambiguity in the direction of the field perpendicular to the line of sight (Metcalf 1994; Metcalf et al. 2006; Leka et al. 2009). In principle vector magnetograms provide boundary conditions for NLFFF modeling, as discussed below.

Vector magnetogram data are now routinely available. In particular satellite observations are provided by the Solar Optical Telescope Spectro-Polarimeter (SOT/SP) on board Hinode (Tsuneta et al. 2008) and the Helioseismic \& Magnetic Imager on the Solar Dynamics Observatory satellite (SDO/HMI) (Scherrer et al. 2012).

\subsection{The model and the boundary conditions}

The NLFFF model for the coronal magnetic field (e.g., Wiegelmann \& Sakurai 2012) is:

$$
\mathbf{J} \times \mathbf{B}=0 \quad \text { and } \quad \nabla \cdot \mathbf{B}=0
$$

where $\mathbf{J}=\mu_{0}^{-1} \nabla \times \mathbf{B}$ is the electric current density, which is everywhere parallel to the magnetic field. Introducing the force-free parameter $\alpha$ via

$$
\mathbf{J}=\alpha \mathbf{B} / \mu_{0},
$$

the equations may be rewritten as:

$$
\mathbf{B} \cdot \nabla \alpha=0 \text { and } \nabla \times \mathbf{B}=\alpha \mathbf{B} .
$$

The boundary conditions for the problem in a half space $z>0$ (e.g., Grad \& Rubin 1958) consist of the values of $B_{z}$ at $z=0$, together with the values of $\alpha$ at $z=0$ over one

$\dagger$ The data presented in DeRosa et al. (2015) are available online, including NLFFF solution cubes for the different methods. See the paper for details. 
polarity of $B_{z}$ (i.e. the region where $B_{z}>0$, here denoted $P$, or the region where $B_{z}<0$, here denoted $N$ ). Values of $J_{z}$ may be prescribed instead of $\alpha$, according to Eq. (2.2).

Some methods of solution of the force-free equations use as boundary conditions the values of $\mathbf{B}$ over both polarities of $B_{z}$, i.e. over both $P$ and $N$. This defines $J_{z}$ over both polarities using

$$
J_{z}=\frac{1}{\mu_{0}}\left(\frac{\partial B_{y}}{\partial x}-\frac{\partial B_{x}}{\partial y}\right)
$$

and is formally an over-prescription.

The methods of solution of Eqs. (2.3) applied to solar data are iterative. These include Grad-Rubin iteration (e.g., Grad \& Rubin 1958; Amari, Boulmezaoud \& Aly 2006; Wheatland 2007), optimization (e.g., Wheatland, Sturrock \& Roumeliotis 2000; Wiegelmann 2007) and the magnetofrictional method (e.g., Valori, Kliem \& Keppens 2005).

In this paper we present NLFFF solutions calculated with a Grad-Rubin code (CFIT). The Grad-Rubin method involves two steps at each iteration. First, currents are run along the field lines of a given field, subject to the boundary conditions on $\alpha$. Second, the field due to this current configuration (and subject to the boundary conditions on the field) is calculated. This provides a new field configuration for the next iteration.

The process of solution of the NLFFF model for given solar boundary data is often referred to as NLFFF 'reconstruction', or 'extrapolation'.

\subsection{The problem of inconsistency and its effects}

Studies comparing NLFFF reconstructions using different methods of solution applied to given vector magnetograms have revealed basic problems (Schrijver et al. 2008; Metcalf et al. 2008; DeRosa et al. 2009; DeRosa et al. 2015). The results may not be accurate solutions to the NLFFF model, for a given solution method, and the results produced by different methods may not agree with one another. In particular they may have substantially different magnetic energies and magnetic free energies, and they may reveal different field line structures.

The problems arise in part from inconsistency between the boundary data and the model. Molodenskii (1969) identified a set of integral identities which must be met in a boundary by a NLFFF, and these identities are generally only approximately satisfied for photospheric vector magnetogram data (e.g., Metcalf et al. 1995; DeRosa et al. 2009). The boundary field values are uncertain due to errors in measurements and field inference (e.g., Leka et al. 2009), but the results also imply non-magnetic forces at the photospheric level (e.g., Metcalf et al. 1995). The NLFFF model may provide a good approximation to magnetic fields in the magnetically-dominated solar corona, at most locations and at most times, but in the denser photosphere forces due to gas pressure, gravity and fluid flows are important. The boundary field is not force free.

The influence of inconsistency on NLFFF modeling depends on the method of solution of the equations. In the following we briefly describe the effects for two popular methods.

\section{Grad-Rubin iteration}

Grad-Rubin methods use the formally correct boundary conditions for the problem, described in $\S 2.2$. However, vector magnetograms permit calculation of $\alpha$ values over both the $P$ and the $N$ polarities (e.g., via Eq. (2.4)). Hence the data provide two sets of boundary conditions and allow calculation of two solutions (the $P$ and the $N$ solutions). For inconsistent boundary data, the two solutions may be substantially different. Also, a Grad-Rubin iteration sequence may not converge. In practice it is difficult to achieve convergence for solar data, and approximate solutions are obtained by a process of trial and error in which the boundary data are modified, e.g., by smoothing $B_{x}$ and $B_{y}$ values 

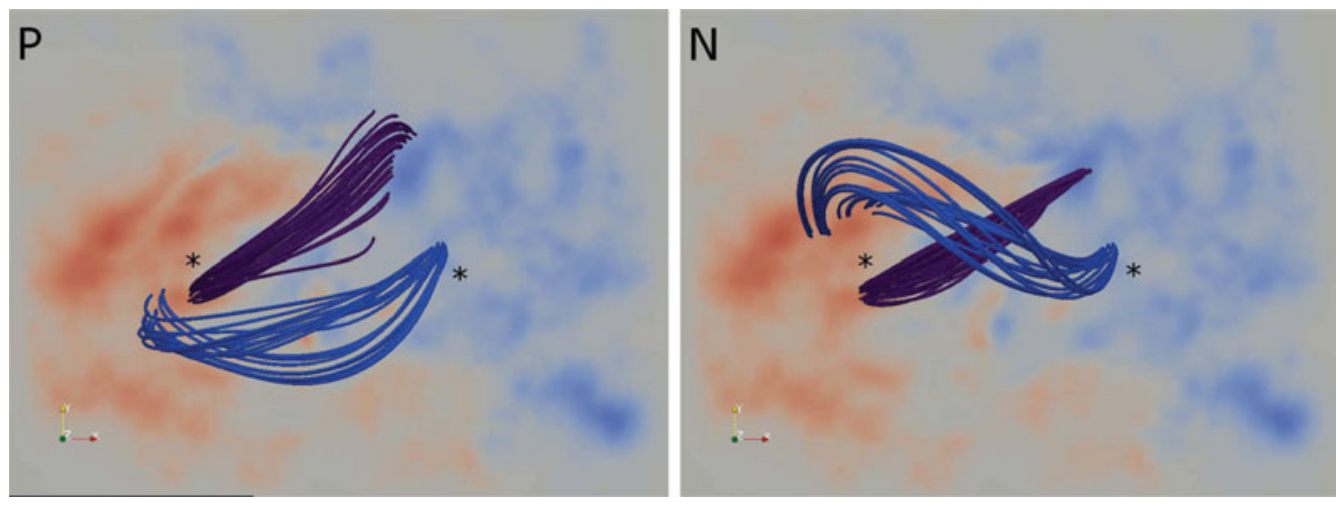

Figure 1. $P$ and $N$ solutions for AR10978 on 12 Dec 2007. The two panels show sets of field lines traced from common starting positions (indicated by the asterisks), for the CFIT bin $4 P$ and $N$ solutions described in DeRosa et al. (2015).

before calculation of $\alpha$ values (e.g., Canou et al. 2009), or by 'censoring' $\alpha$ values, i.e. assigning $\alpha=0$ in weak field regions (e.g., Wheatland \& Leka 2011; Amari, Canou \& Aly 2014).

Figure 1 illustrates the effects. The figures shows two of the CFIT solutions discussed in DeRosa et al. (2009), which are constructed for a vector magnetogram based on Hinode SOT/SP data for active region AR10978 observed on 12 December 2007. The two panels show the CFIT bin $4 P$ solution (left) and the CFIT bin $4 N$ solution (right). The views look down vertically on the computational domain, and the red and blue in the background indicates positive and negative values of the vertical field $B_{z}$. The blue and purple curves are sets of field lines originating from sets of points in the lower boundary (the starting positions are indicated by asterisks). The purple field lines appear similar for the $P$ and $N$ solutions, but the blue field lines are qualitatively different. For these solutions values of $\alpha$ are censored to achieve approximate convergence of the Grad-Rubin iteration sequence. The free energies of the $P$ and $N$ solutions are similar: $E_{P} / E_{P, 0}=1.11$ and $E_{N} / E_{N, 0}=1.10$, where $E_{P, 0}$ and $E_{N, 0}$ are the reference potential field energies.

Optimization

Optimization (e.g., Wheatland, Sturrock \& Roumeliotis 2000; Wiegelmann 2007) uses values of $\mathbf{B}$ over both $P$ and $N$ as boundary conditions. In principle there is only one result, but for inconsistent boundary conditions the result cannot be an accurate solution to the model. The result may have $\mathbf{J} \times \mathbf{B} \neq 0$ and/or $\nabla \cdot \mathbf{B} \neq 0$. (The optimization method uses initial conditions with explicit departure from the 'solenoidal' state $\nabla \cdot \mathbf{B}=0$. If an accurate solution to the model is obtained, it is close to solenoidal.)

Preprocessing is often applied to vector magnetogram data prior to use of optimization (Wiegelmann, Inhester \& Sakurai 2006; Wiegelmann \& Inhester 2010). In this procedure the field boundary values are altered to better satisfy the Molodenskii (1969) conditions. In practice preprocessing is found to improve the quality of NLFFF solutions based on some metrics. However, the Molodenskii integrals represent necessary (but not sufficient) conditions for the existence of a NLFFF, and preprocessed boundary conditions are in general still inconsistent with the model (e.g., DeRosa et al. 2009).

\subsection{Changes in the boundary conditions}

As $\oint 2.3$ suggests, it is generally necessary to change the boundary values provided by a vector magnetogram to achieve a satisfactory solution to the NLFFF model. 


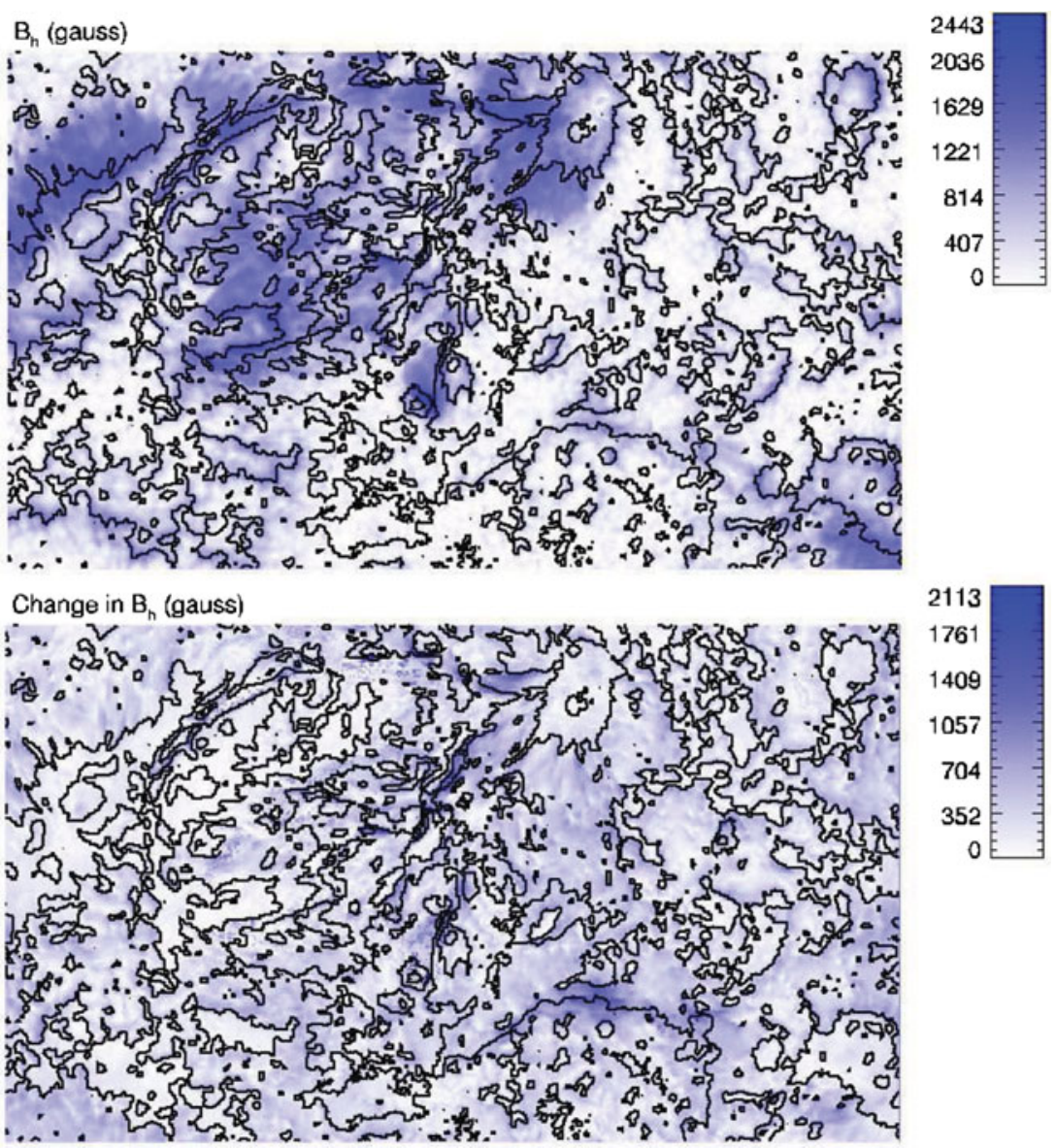

Figure 2. Changes in the boundary conditions for the horizontal field of AR10978 (by comparison with the vector magnetogram) for the CFIT bin $2 P$ solution (DeRosa et al. 2015). The top panel shows the magnitude of the field, and the bottom panel shows the magnitude of the change.

Figure 2 illustrates the size of the changes required for one of the solutions in DeRosa et al. (2015). The figure shows the changes in the boundary values of the horizontal field $B_{h}=\sqrt{B_{x}^{2}+B_{y}^{2}}$ for the CFIT $P$ solution at the bin 2 resolution. (The Grad-Rubin method does not change the vertical component of the field.) The top panel shows the magnitude of the horizontal field, and the bottom panel shows the magnitude of the change in the horizontal field. The figure indicates that there are substantial changes in $B_{h}$ across the boundary region, and that the maximum change is comparable to the maximum value of $B_{h}$. DeRosa et al. (2015) present a more detailed investigation of the changes in the boundary field introduced by different solution methods, and a comparison of the changes between methods.

\subsection{Assessing the errors in solutions}

In assessing the result of a NLFFF reconstruction, it is useful to consider two (related) questions:

- Q1 - Does it represent what is on the Sun?

- Q2 - Is it an accurate solution to the model? 
Approaches to answer Q1 may include comparison of field line traces with EUV/X-ray images, comparison with results obtained between NLFFF methods, or with other models (e.g., MHD), and consideration of the changes in the boundary conditions required to achieve a solution ( $\operatorname{see} \S 2.4)$.

Q2 is often addressed by calculating two specific metrics (Wheatland, Sturrock \& Roumeliotis 2000). The pointwise average of $\left|\nabla \cdot \mathbf{B}_{i}\right|$ over grid points $i$ provides a measure of the solenoidal error, and a weighted average angle between $\mathbf{J}_{i}$ and $\mathbf{B}_{i}$ over grid points is presented as an indicator of magnetic forces. However these 'answers' to Q2 are difficult to interpret, and are unrelated to how the solutions are used. For example, NLFFF solutions are often used to estimate magnetic energy or magnetic free energy, but these metrics do not indicate whether the resulting energy estimates are reliable.

\section{Recommendations}

Based on the results in DeRosa et al. (2015), NLFFF modeling of coronal magnetic fields for active regions remains challenging. Codes may produce solutions of varying quality, and a range of results may be obtained for the same vector magnetogram.

Here we make two specific, simple recommendations for answering Q2 (checking the quality of the solution to the model), based on the intended use of the solution.

First, if solutions are being used to estimate magnetic energy, we recommend using the method of Valori et al. (2013) to calculate the non-solenoidal contributions to the energy, following DeRosa et al. (2015). Valori et al. (2013) show how to decompose the field into potential $(p)$ and current carrying $(c)$ components, each with solenoidal $(s)$ and non-solenoidal (ns) parts. The total magnetic energy $E$ may be expressed as

$$
E=E_{p, s}+E_{p, n s}+E_{c, s}+E_{c, n s}+E_{\text {mix }},
$$

where

$$
\begin{aligned}
& E_{p, s}=\frac{1}{2 \mu_{0}} \int \mathbf{B}_{p, s}^{2} d V, \quad E_{p, n s}=\frac{1}{2 \mu_{0}} \int \mathbf{B}_{p, n s}^{2} d V \\
& E_{c, s}=\frac{1}{2 \mu_{0}} \int \mathbf{B}_{c, s}^{2} d V, \quad E_{c, n s}=\frac{1}{2 \mu_{0}} \int \mathbf{B}_{c, n s}^{2} d V
\end{aligned}
$$

and

$$
\begin{aligned}
E_{\mathrm{mix}}=\frac{1}{\mu_{0}} \int & \left(\mathbf{B}_{p, s} \cdot \mathbf{B}_{p, n s}+\mathbf{B}_{c, s} \cdot \mathbf{B}_{c, n s}+\mathbf{B}_{p, s} \cdot \mathbf{B}_{c, n s}\right. \\
& \left.+\mathbf{B}_{c, s} \cdot \mathbf{B}_{p, n s}+\mathbf{B}_{p, n s} \cdot \mathbf{B}_{c, n s}+\mathbf{B}_{p, s} \cdot \mathbf{B}_{c, s}\right) d V .
\end{aligned}
$$

For a solenoidal field $E=E_{p}+E_{c}$ with $E_{p}=E_{p, s}$ and $E_{c}=E_{c, s}$, and

$$
E_{p, n s}=E_{c, n s}=E_{\text {mix }}=0 .
$$

For a NLFFF solution it is necessary to check that $E_{p, n s}, E_{c, n s}$ and $\left|E_{\text {mix }}\right|$ are small compared with the magnetic free energy, which is the component $E_{c, n s}$. The results in DeRosa et al. (2015) suggest that solutions obtained by the optimization method, in particular, may fail this test.

Second, if solutions are being used to identify specific field structures, then we recommend tracing relevant field lines, and current streamlines (field lines of $\nabla \times \mathbf{B}$ ) from common starting points. If the field is force free, then the field lines and current streamlines should agree. This provides a stringent test because departures in the two paths add up along the paths. In general the sets of field lines and current streamlines will not be 


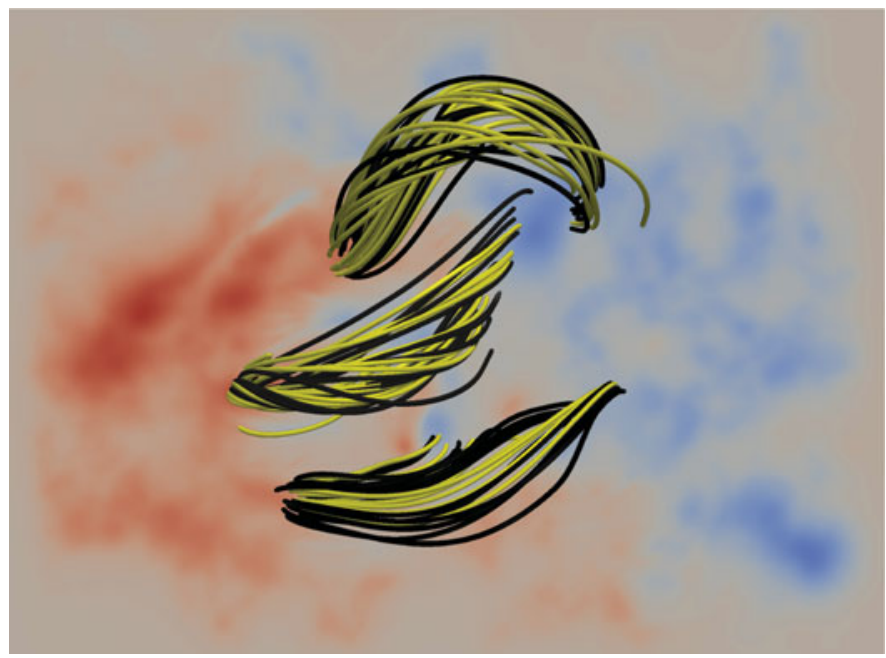

Figure 3. Traces of field lines (black) and current streamlines (yellow) for three sets of common starting points, for the CFIT bin 4 P solution for AR10978 described in DeRosa et al. (2015).

exactly coincident, but they should both reproduce structures of interest (e.g., 'twisted flux ropes').

Figure 3 illustrates the second recommended test, for one of the CFIT solutions in DeRosa et al. (2015), namely the $\mathrm{P}$ solution at the bin 4 resolution. The figure shows three bundles of field lines (black curves), and corresponding current streamlines (yellow curves). The three sets of curves do not agree exactly but are qualitatively similar. The departures occur because the field is only approximately force free.

\section{Summary}

This paper presents a short review of nonlinear force-free field (NLFFF) modeling of coronal magnetic fields in active regions. Coronal field modeling is often motivated by solar activity, and the role of activity in space weather.

Vector magnetograms provide boundary values for coronal field 'extrapolation' based on the nonlinear force-free field model, and this modeling approach has become quite popular. However, studies suggest that the results may be unreliable. In particular, the inconsistency of the solar data with the NLFFF model is the source of problems, which depend in detail on the method of solution of the model.

We recommend two simple tests for NLFFF models, which assess the quality of the solutions. The tests are chosen to match the use made of the NLFFF solutions.

\section{References}

Amari, T., Boulmezaoud, T. Z., \& and Aly, J. J. 2006, Astron. Astroph. 446, 691-705, doi: 10.1051/0004-6361:20054076

Amari, T., Canou, A., \& Aly, J.-J. 2014, Nature 514, 466-469, doi: 10.1038/nature13815

Baker, D. N. et al. 2008, Severe Space Weather Events: Understanding Societal and Economic Impacts Workshop Report, National Academies Press: Washington DC, doi: 10.17226/12507

Canou, A., Amari, T., Bommier, V., Schmieder, B., Aulanier, G., \& Li, H. 2009, Astrophys. J. 693, L27-L30, doi: 10.1088/0004-637X/693/1/L27

del Toro Iniesta, J. C.: 2003, Introduction to Spectropolarimetry, Cambridge University Press, Cambridge, UK, ISBN: 0521818273 
DeRosa, M. L. et al. 2009, Astrophys. J. 696, 1780-1791, doi: 10.1088/0004-637X/696/2/1780

DeRosa, M. L. et al. 2015, Astrophys. J. 811, 107 (21pp) doi: 10.1088/0004-637X/811/2/107

Gilchrist, S. A. \& Wheatland, M. S. 2013, Sol. Phys. 282, 283-302, doi: 10.1007/s11207-0120144-0

Grad, H. \& Rubin, H. 1958, in Proc. 2nd Int. Conf. on Peaceful Uses of Atomic Energy, UN: Geneva, 190-197

Leka, K. D. et al. 2009, Solar Phys. 260, 83-108, doi: 10.1007/s11207-009-9440-8

Metcalf, T. R. 1994, Solar Phys. 155, 235-242, doi: 10.1007/BF00680593

Metcalf, T. R., Jiao, L., McClymont, A. N., Canfield, R. C., \& Uitenbroek, H. 1995, Astrophys. J. 439, 474-481, doi: 10.1086/175188

Metcalf, T. R. et al. 2006, Solar Phys. 237, 267-296, doi: 10.1007/s11207-006-0170-x

Metcalf, T. R. et al. 2008, Solar Phys. 247, 269-299, doi: 10.1007/s11207-007-9110-7

Molodenskii, M. M. 1969, Soviet Astronomy 12, 585-588

Scherrer, P. H. et al. 2012, Solar Phys. 275, 207, doi: 10.1007/s11207-011-9834-2

Schrijver, C. J. et al. 2008, Astrophys. J. 675, 1637-1644, doi: 10.1086/527413

Tsuneta, S. et al. 2008, Solar Phys. 249, 167-196, doi: 10.1007/s11207-008-9174-z

Valori, G., Démoulin, P., Pariat, E., \& and Masson, S. 2013, Astron. Astroph. 553, A38 (14pp), doi: 10.1051/0004-6361/201220982

Valori, G., Kliem, B., \& Keppens, R. 2005, Astron. Astroph. 433, 335-347, doi: 10.1051/00046361:20042008

Wheatland, M. S. 2007, Solar Phys. 245, 251-262, doi: 10.1007/s11207-007-9054-y

Wheatland, M. S. \& Leka, K. D. 2011, Astrophys. J. 728, 112 (12pp), doi: 10.1088/0004$637 \mathrm{X} / 728 / 2 / 112$

Wheatland, M. S., Sturrock, P. A., \& Roumeliotis, G. 2000, Astrophys. J. 540, 1150-1155s, doi: $10.1086 / 309355$

Wiegelmann, T. 2007, Solar Phys. 240, 227-239, doi: 10.1007/s11207-006-0266-3

Wiegelmann, T. \& Inhester, B. 2010, Astron. Astroph. 516, A107 (5pp), doi: 10.1051/0004$6361 / 201014391$

Wiegelmann, T., Inhester, B., \& Sakurai, T. 2006, Solar Phys. 233, 215-232, doi: 10.1007/s11207006-2092-z

Wiegelmann, T., Petrie, G. J. D., \& Riley, P. 2015, Space Sci. Rev., 1-26, Open Access, doi: $10.1007 / \mathrm{s} 11214-015-0178-3$

Wiegelmann, T. \& Sakurai, T. 2012, Living Reviews in Solar Physics 9, doi: 10.12942/lrsp-2012-5 